\title{
Effect of porcine immature oocyte vitrification on oocyte-cumulus cell gap junctional intercellular communication
}

\author{
Fahiel Casillas ${ }^{1 *} \mathbb{D}$, Yvonne Ducolomb ${ }^{2 \wedge}$, Alma López $^{2}$ and Miguel Betancourt ${ }^{2}$
}

\begin{abstract}
Vitrification may severely affect cumulus cells and oocyte morphology and viability, limiting their maturation and developmental potential. The aim of this study was to evaluate the gap junction intercellular communication (GJIC) integrity after the vitrification of porcine immature cumulus-oocyte complexes (COCs). Fresh COCs were randomly distributed in three groups: untreated (control), toxicity (cryoprotectants exposure), and vitrification, then subjected to in vitro maturation (IVM). Oocyte viability and IVM were measured in all groups. The evaluation of GJIC was expressed as relative fluorescence intensity (RFI). Vitrification significantly decreased oocyte viability and maturation after $44 \mathrm{~h}$ of culture compared to control. Also, significantly reduced RFI was observed in vitrified COCs during the first hours of culture (4-8 h) compared to control. This study demonstrates that porcine oocyte viability and maturation after $44 \mathrm{~h}$ of culture decreased after vitrification. GJIC was also affected during the first hours of culture after the vitrification of immature oocytes, being one of the possible mechanisms by which oocyte maturation decreased.
\end{abstract}

Keywords: Vitrification, Porcine, Oocyte, Viability, Maturation, Gap junctions

\section{Introduction}

Oocyte cryopreservation has enabled significant advances in fertility treatments in humans and allows the genetic improvement of livestock. It has been reported that vitrification may severely affect cumulus cells (CCs) and oocyte morphology and viability, limiting their maturation and developmental potential [1]. Premature meiotic progression [2], low maturation [3, 4], and embryo development rates $[5,6]$ have been reported after the vitrification of porcine germinal vesicle (GV) immature oocytes. So far, some studies have reported the birth of live offspring from vitrified GV oocytes [7], and porcine embryos $[8,9]$.

\footnotetext{
* Correspondence: fahiel@xanum.uam.mx

Yvonne Ducolomb is deceased

'Departamento de Biología de la Reproducción, División de Ciencias Biológicas y de la Salud, Universidad Autónoma Metropolitana-Iztapalapa, 09340 CDMX, México

Full list of author information is available at the end of the article
}

Oocyte growth is related to the functionality of the somatic cells surrounding the oocyte, which are known as granulosa cells. These cells provide the oocyte essential nutrients for later stages: maturation, fertilization, and embryo development [10]. CCs primary function is to regulate the mechanism of oocyte meiotic arrest and resumption [11]. Maintaining the viability of the CCs is critical for oocyte maturation after vitrification. It has been reported that CCs viability is affected after vitrification [3], reducing oocyte maturation. Different types of cell communication have been described, such as tight junctions, desmosomes, cell adhesion molecules, and gap junction intercellular communication (GJIC) [12]. The latter establishes communication between CCs-oocyte and consists of arrays of intercellular channels that allow the exchange by passive diffusion of compounds $<1$ $\mathrm{KDa}$ as metabolites, ions, sugars, second messengers, and water [13]. Gap junctions are composed of 
membrane hemichannels called connexons, each of them composed of six transmembrane protein subunits called connexins. GJIC is directly involved in oocyte meiotic arrest and resumption providing a direct communication by which cGMP enters the oocyte, inhibiting phosphodiesterase A, maintaining high levels of cAMP, and the meiotic arrest [14]. For resumption, LH receptor activation in the CCs reduces the influx of cGMP by causing the GJIC closure and meiotic resumption in mouse [15] and pigs [14]. Previous studies indicate that the integrity of the GJIC between CCs-oocyte during the first $4 \mathrm{~h}$ of maturation is crucial to resume meiosis properly $[14,16-18]$. Also, GJIC is important for the regulation of chromatin remodeling and transcription during the first hours of bovine oocyte maturation [19]. The knowledge of the possible mechanisms involved in reducing GV oocyte maturation after vitrification is important for the development of more efficient vitrification protocols. Therefore, the aim of this study was to evaluate the GIIC integrity after the vitrification of immature porcine cumulus-oocyte complexes (COCs).

\section{Experimental design}

Fresh COCs were randomly distributed in three groups: untreated (control), toxicity (cryoprotectants exposure), and vitrification, then subjected to in vitro maturation (IVM). Before and after IVM, at $0 \mathrm{~h}$ and $44 \mathrm{~h}$, oocyte viability was measured in all groups. For viability, at least three replicates were performed with $n=162$ control, $n=107$ toxicity, and $n=128$ vitrification, evaluated oocytes. For maturation, seven experiments were performed with $n=250$ control, $n=175$ toxicity, and $n=175$ vitrification, evaluated oocytes. GJIC integrity was evaluated at 4, 8, 22, and $44 \mathrm{~h}$ of culture. Experiments were carried out in triplicate with $n=80$ control, $n=80$ carbenoxolone (positive control), $n=60$ toxicity, and $n=80$ vitrification, evaluated oocytes.

\section{Methods}

Unless otherwise mentioned, all chemicals were acquired from Sigma Chemical Co. (St. Louis, MO, USA). For oocyte collection and IVM, ovaries were obtained from pre-pubertal Landrace gilts at a local slaughterhouse "Los Arcos", located in Texcoco, State of Mexico (animal health federal law authorization 6265375) and transported to the laboratory in a $0.9 \% \mathrm{NaCl}$ solution at $25^{\circ} \mathrm{C}$ within $2 \mathrm{~h}$ after collection. Ovarian follicles between 3 and $6 \mathrm{~mm}$ in diameter were punctured to obtain COCs. For COCs collection and washing, Tyrode modified medium supplemented with $10 \mathrm{mM}$ sodium lactate, 10 $\mathrm{mM}$ HEPES and $1 \mathrm{mg} / \mathrm{mL}$ polyvinyl alcohol (PVA) (TLHEPES-PVA) was used. Only oocytes with uniform cytoplasm surrounded by a two-four-layer compact mass of CCs were selected. COCs were washed three times in $500 \mu \mathrm{L}$ drops of maturation medium composed of TCM199 with Earle's salts and $26.2 \mathrm{mM}$ sodium bicarbonate (In Vitro, Mexico City) and supplemented with 0.1\% PVA, $3.05 \mathrm{mM}$ D-glucose, $0.91 \mathrm{mM}$ sodium pyruvate, $0.57 \mathrm{mM}$ cysteine and $10 \mathrm{ng} / \mathrm{mL}$ EGF. For IVM, groups from 30 to 50 COCs were placed in each well of a fourwell dish (Thermo-Scientific Nunc, Rochester NY) containing $500 \mu \mathrm{L}$ of maturation medium supplemented with $0.5 \mu \mathrm{g} / \mathrm{mL}$ luteinizing hormone (LH) and $0.5 \mu \mathrm{g} / \mathrm{mL}$ follicle stimulating hormone (FSH) for $44 \mathrm{~h}$ [5]. Oocyte culture was performed under mineral oil and incubated at $38.5{ }^{\circ} \mathrm{C}$ in an atmosphere of $5 \% \mathrm{CO}_{2}$ in air and humidity at saturation.

For COCs exposure to cryoprotectants before IVM, groups of eight to ten COCs were exposed to the highest CPAs concentration solution containing TCM-199, $16 \%$ dimethylsulphoxide $\left(\mathrm{Me}_{2} \mathrm{SO}\right), 16 \%$ ethylene glycol (EG) and $0.4 \mathrm{M}$ sucrose at $38.5^{\circ} \mathrm{C}$ for $1 \mathrm{~min}$ (toxicity group). Immediately, $\mathrm{COCs}$ were recovered and washed three times in TL-HEPES-PVA medium and transferred to TCM-199 medium for IVM. Exposure time and temperature were selected to make them comparable to values commonly used for oocyte vitrification protocols [5].

For COCs vitrification and warming, the medium used was TCM-199-HEPES supplemented with $0.5 \mathrm{mM} \mathrm{L-glu-}$ tamine and $0.1 \%$ PVA (VW medium). For vitrification, COCs were washed twice in VW medium and sequentially equilibrated in the first vitrification solution containing 7.5\% $\mathrm{Me}_{2} \mathrm{SO}+7.5 \% \mathrm{EG}$ for $3 \mathrm{~min}$, and $1 \mathrm{~min}$ in a second vitrification solution containing $16 \% \mathrm{Me}_{2} \mathrm{SO}$, $16 \% \mathrm{EG}$ and $0.4 \mathrm{M}$ sucrose. Then, groups of seven COCs were placed in a $2 \mu \mathrm{L}$ drop of the second vitrification solution and loaded into the Cryolock device (Importadora Mexicana de Materiales para Reproduccion Asistida S. A. de C.V., Mexico) in less than $1 \mathrm{~min}$. Then, the Cryolock device was immediately plunged horizontally into liquid nitrogen and stored during $30 \mathrm{~min}$ [5]. Warming was performed by the one-step method [20]. For COCs recovery, the Cryolock device was submerged vertically in a four-well dish containing $800 \mu \mathrm{L}$ of VW medium supplemented with $0.13 \mathrm{M}$ sucrose. Finally, COCs were incubated in VW medium for $5 \mathrm{~min}$, then recovered and transferred to maturation medium.

Oocyte viability was measured at $\mathrm{T} 0 \mathrm{~h}=$ immediately after COCs collection, and at T $44 \mathrm{~h}=$ after IVM in all groups. Oocytes were stained with $100 \mu \mathrm{L}$ of $0.5 \mathrm{mg} / \mathrm{mL}$ Thiazolyl blue (MTT) diluted in PBS. The MTT stain evaluates the metabolic activity of cells. NADPHdependent cellular oxidoreductase enzymes are capable of reducing the tetrazolium dye MTT to formazan (reflecting purple coloration) as an indicator of viable 
cells. After $1 \mathrm{~h}$, oocytes were analyzed under a light microscope (Zeiss Axiostar) and classified as viable (purple-stained) and non-viable (colorless). To evaluate IVM, oocytes were stained with $10 \mu \mathrm{g} / \mathrm{mL}$ bisbenzimide (Hoechst 33342) diluted in PBS for $45 \mathrm{~min}$ and evaluated using an epifluorescence microscope (Zeiss Axiostar) at $40 \mathrm{X}$ magnification. Oocytes showing a GV, or in the metaphase I (MI) were considered immature, and those in the metaphase II (MII) with the first polar body as matured. After COCs cryoprotectants exposure and vitrification, GIC transfer between CCs-oocyte was evaluated using the acetoxymethyl (AM) ester derivative of the fluorescent indicator calcein (calcein-AM; Molecular Probes, Eugene, OR) method [14]. After 4, 8, 22 and 44 $\mathrm{h}$ of culture, COCs from all groups were exposed to calcein-AM for $15 \mathrm{~min}$, then were transferred to calceinAM free media and cultured for $25 \mathrm{~min}$, allowing the dye exchange between CCs-oocyte. In the oocyte cytoplasm, nonspecific endogenous esterases hydrolyze the acetoxy-methyl groups to produce calcein (MW 622.54), a negatively charged molecule that is unable to permeate through the plasma membrane and can only pass from the CCs to the oocyte cytoplasm through GJIC, producing a fluorescent green coloration. Then, COCs were washed once in $0.01 \%(\mathrm{w} / \mathrm{v})$ PVA and PBS and were analyzed under a confocal epifluorescence microscope (Zeiss, LSM T-PMT) with an argon laser $(488 \mathrm{~nm})$ at 60 $\mathrm{X}$ magnification to obtain pictures. Fluorescence intensity (FI) profiles of calcein in CCs-oocytes were obtained using the Zen blue lite 2.3 software. Lines were drawn through the COCs optical sections generated by the confocal microscope. Carbenoxolone, a gap junction blocker, was used at $100 \mu \mathrm{M}$ as a positive control [21].

\section{Statistical analysis}

Statistical analyses were carried out using GraphPad Prism 8.2.1 (GraphPad Software Inc.). The percentage of oocyte viability, maturation, and FI in control, toxicity, and vitrification groups were analyzed by a one-way ANOVA followed by Bonferroni multiple comparison test with a confidence level of $P<0.05$. Data are presented as mean $\pm \mathrm{SD}$.

\section{Results}

Oocyte viability at $\mathrm{T} 0 \mathrm{~h}$ was not statistically different from control $(P>0.05)$. However, cryoprotectants exposure and vitrification significantly decreased oocyte viability after $44 \mathrm{~h}$ of culture compared to control $(P<0.05)$, as shown in Fig. 1a. After vitrification, the MI stage was not affected compared to control. However, a significantly high percentage of GV oocytes was observed after cryoprotectants exposure and vitrification compared to control. Consequently, the percentage of MII oocytes significantly decreased after cryoprotectants exposure and vitrification compared to control. Also, the percentage of oocyte maturation was significantly lower in the vitrification group compared to the toxicity group (Fig. 1b). The evaluation of GIIC was performed after 4, 8, 22, and $44 \mathrm{~h}$ of culture, and it was expressed as relative fluorescence intensity (RFI), as shown in Fig. 2. In control, significantly high RFI was observed at 4 and $8 \mathrm{~h}$, which was decreased at 22 and $44 \mathrm{~h}$. After cryoprotectants exposure and vitrification, significantly reduced RFI was observed in COCs at 4 and $8 \mathrm{~h}$ compared to control. At 22 and $44 \mathrm{~h}$, reduced or absent RFI was observed in all groups. Also significantly reduced RFI was observed in the vitrification group compared to the toxicity group (Fig. 2). Representative images of GIC evaluation after vitrification are shown in Fig. 3. CCs-oocyte GJIC was measured by the quantitative FI profiles of calcein in COCs (Fig. 3a). In control, results showed that high FI is observed in the CCs-oocyte at 4 and $8 \mathrm{~h}$ (red lines). In the carbenoxolone group (a gap junction blocker), high FI was observed in the CCs but not in the oocyte at 4 and $8 \mathrm{~h}$ (red crossed lines). In the toxicity and vitrification groups, reduced FI was observed in the CCs-oocyte at 4 and $8 \mathrm{~h}$. Also, in control and vitrification groups, reduced FI was observed in CCs-oocyte at 22 and $44 \mathrm{~h}$ (Fig. 3a). For all groups, FI profiles were measured in COCs using the confocal microscope software, as shown in Fig. 3b.

\section{Discussion and conclusion}

The present study demonstrates that porcine immature oocyte viability decreased after $44 \mathrm{~h}$ of culture. These results confirm those previously reported [22], where the viability of pig oocytes decreases after IVM. This could be explained by the fact that during in vitro culture, the levels of $\mathrm{O}_{2}$ [23] and reactive oxygen species increase [24], affecting cell viability. Likewise, in the GV stage, oocytes are highly cryo-sensitive during the vitrification process compared to other developmental stages. Porcine oocytes vitrified in the GV stage, present a high intracellular lipid content, which prevents the permeability and protection capacity of intracellular cryoprotectants. Therefore, a high amount of intracellular lipids affects glutathione levels in vitrified oocytes, which increases the production of $\mathrm{H}_{2} \mathrm{O}_{2}$ and the generation of oxidative stress [25]. Also, GV oocyte meiotic spindle organization, chromosome and actin filaments distribution has been reported to be extremely sensitive to low temperatures, compared with MII oocytes [26]. Our results demonstrate that oocyte maturation was significantly affected after vitrification. However, the percentage of MI oocytes was not different. This may be due to the fact that some oocytes were able to break the GV, but remained arrested in MI without progressing to MII, 


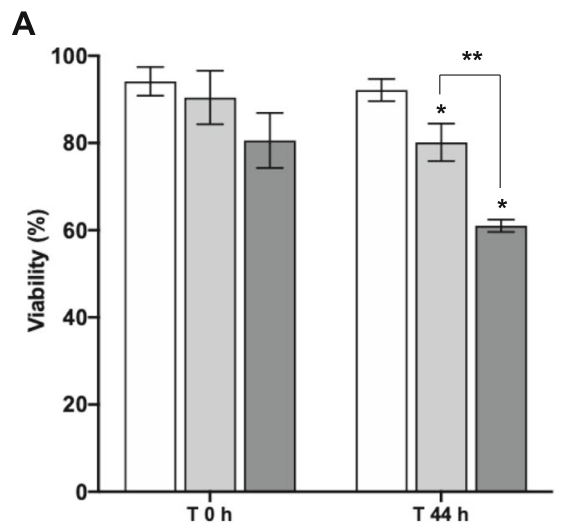

B

$\square$ Control

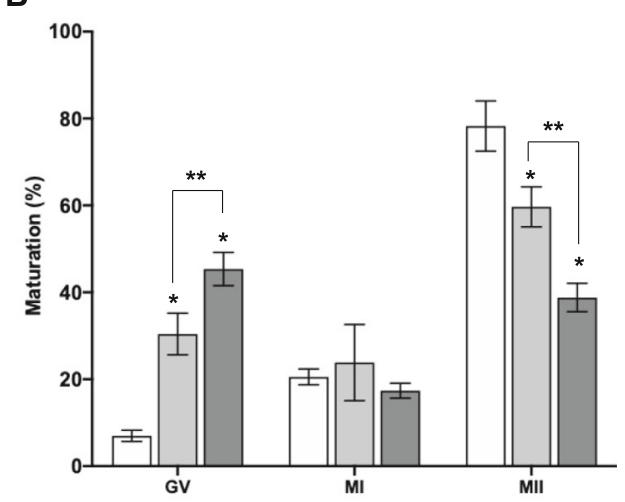

Toxicity

Vitrification

Fig. 1 Effect of vitrification on oocyte viability and in vitro maturation. a Percentage of oocyte viability at $\mathrm{T} 0 \mathrm{~h}$ (immediately after vitrification) and after $44 \mathrm{~h}$ of culture. Oocyte viability decreased after $44 \mathrm{~h}$ of culture in the toxicity and vitrification groups. $\mathbf{b}$ Percentage of oocytes in different developmental stages after $44 \mathrm{~h}$ of culture. Oocyte maturation (MII stage) decreased in toxicity and vitrification groups. $\mathrm{GV}=$ germinal vesicle; $\mathrm{Ml}=$ metaphase I; MII = metaphase II. Bars show the mean \pm SD. * Significant differences vs. control. ** Significant differences toxicity vs. vitrification

where a significant decrease in oocyte maturation was observed.

GJIC is directly involved in oocyte meiotic arrest and resumption. In the present study, we evaluated the integrity of GJIC after vitrification using the calcein-AM transfer assay. As far as we know, information about the integrity of GIC after the vitrification of porcine GV oocytes is limited. In porcine, using transmission electron microscopy, a study reported that gap junctions and microvilli were ruptured after GV oocyte vitrification [27]. Another study evaluated the transzonal projections (TZPs), by marking F-actin, as an indicator of gap junctions integrity. They observed the formation of large clusters of intracellular F-actin in vitrified oocytes [2]. Also, in feline GV oocytes, GIIC was evaluated by the lucifer yellow dye microinjection. They reported that vitrification by slow freezing or vitrification impairs intercellular junctions, which resulted in low oocyte

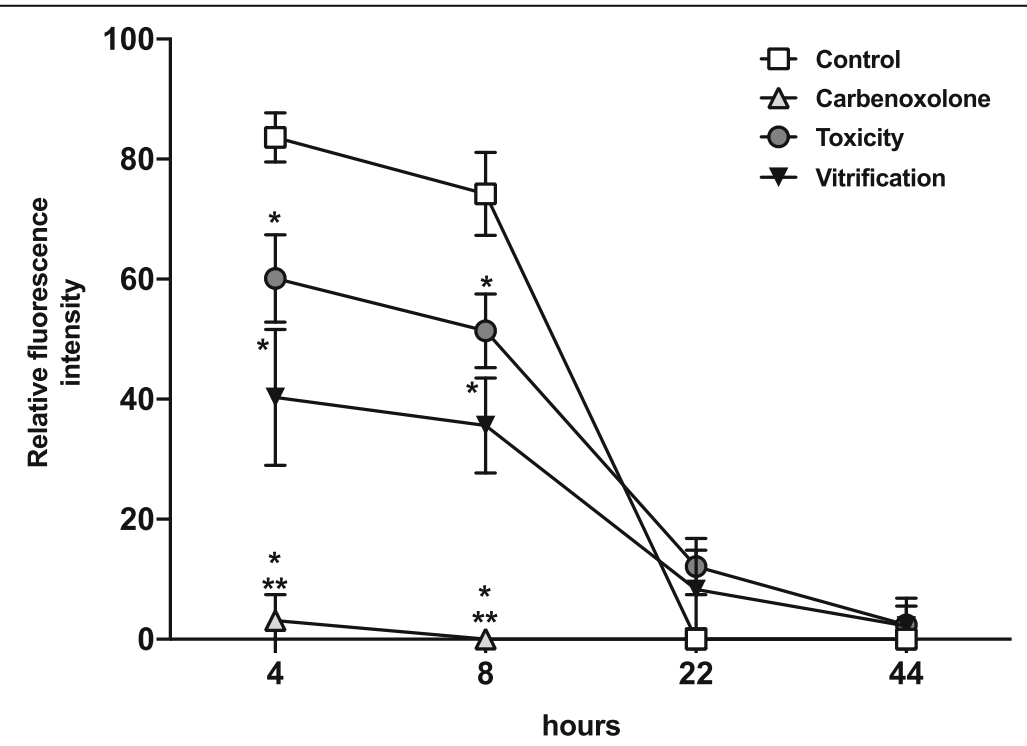

Fig. 2 Effect of vitrification on GJC in COCs during in vitro maturation. GJIC was measured in COCs after 4, 8, 22, and 44 h of culture by quantitative RFI. Lines were drawn through the COCs optical sections generated by the confocal microscope. Carbenoxolone was used as a positive control of GJIC blockage. The RFI values were converted to a scale of 1-100 for graphic representation. After cryoprotectants exposure (toxicity) and vitrification, GJIC decreased at 4 and $8 \mathrm{~h}$ of culture. $\mathrm{GJIC}=$ gap junction intercellular communication; COCs = cumulus-oocyte complexes; RFI = relative fluorescence intensity. Bars show the mean \pm SD. * Significant differences vs. control. ** Significant differences vitrification vs. carbenoxolone 


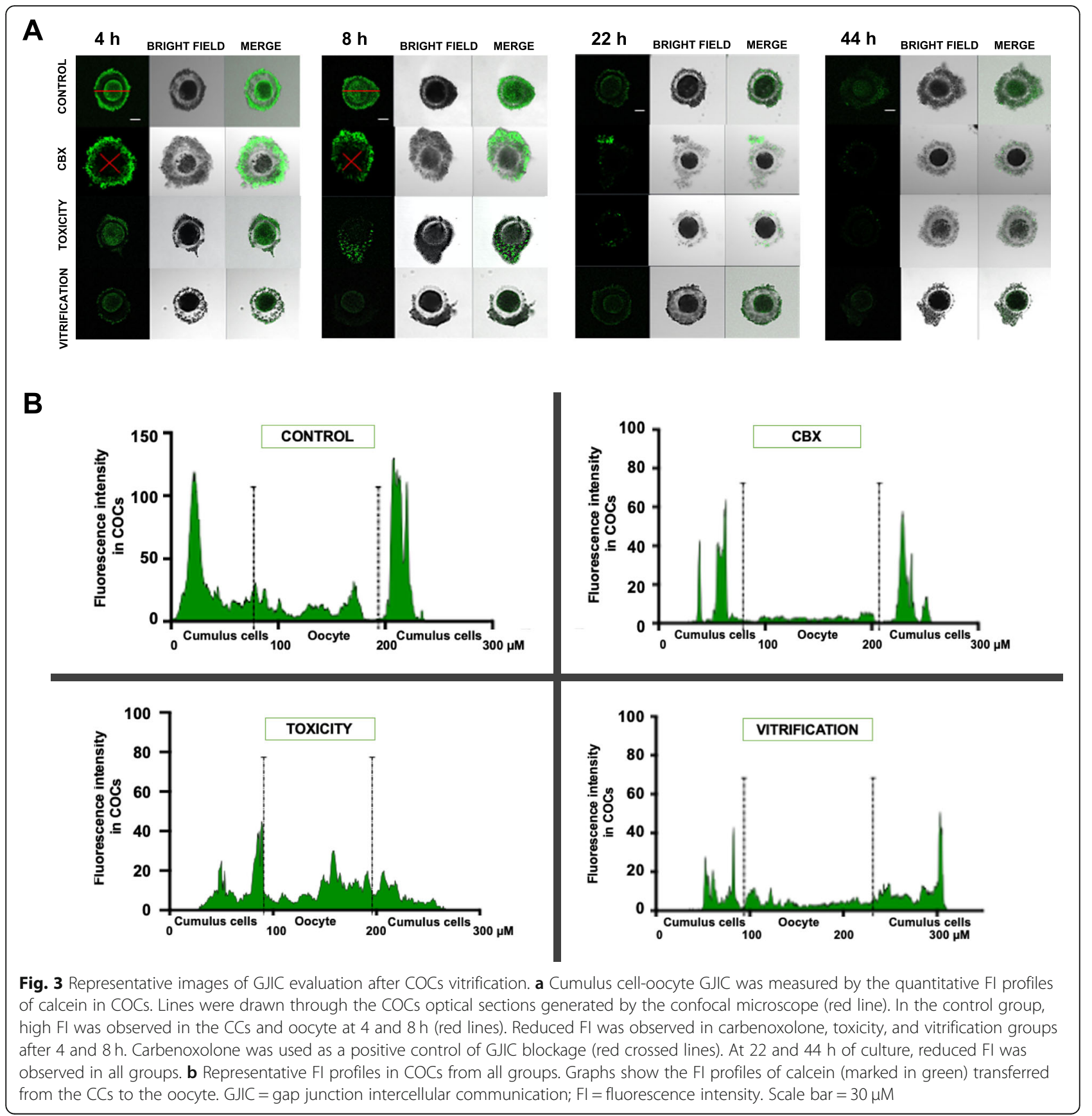

maturation (32.5 and 14.1\%, respectively) [28]. It has been reported that during the first hours of maturation (4-8 h) the functionality of the GIIC between the CCsoocyte is crucial for the mechanism of meiotic resumption [14]. Domínguez et al. (2019) [18] reported that porcine oocyte exposure to perfluorodecanoic acid (PFDA) affected GIC at $4.5 \mathrm{~h}$ of IVM suggesting that the inhibition of oocyte maturation by PFDA might be explained by the disruption of GJIC during the first $18 \mathrm{~h}$ of IVM. In the present study, during the first hours of culture, vitrified oocytes displayed lower FI, affecting
GJIC. It was also observed that the functionality of the GIC decreased from 22 to $44 \mathrm{~h}$ of IVM in the control. Appeltant et al. (2017) [2] reported that at $0 \mathrm{~h}$ the TZPs are present but at $20 \mathrm{~h}$ they are no longer observed. Some studies point out the possible relationship between the disruption of the GJIC during the first hours of culture with a decrease in IVM rates [17, 18]. It has been reported that through gap junctions, metabolites and signaling molecules are exchanged between the cumulus cells and the oocyte during the first $18 \mathrm{~h}$, and subsequently decrease producing the rupture of the GV [14]. 
It has also been reported that cell communication between these cells during the first hours of IVM in bovine oocytes plays an important role in the regulation of chromatin remodeling and transcription [19].

Our results showed that vitrification impairs GJIC between the CCs-oocyte, which could explain the low rates of IVM obtained. GJIC functionality was observed in control oocytes at 4 and $8 \mathrm{~h}$, in order to promote meiotic resumption. It was reported that GJIC functionality is critical during the first $4.5 \mathrm{~h}$ of IVM for meiotic resumption [16-18]. However, GJIC was significantly compromised after vitrification compared to control. Therefore, this study demonstrates that porcine oocyte viability and maturation after $44 \mathrm{~h}$ of culture decreased after vitrification. GJIC was also affected during the first hours of culture after the vitrification of immature oocytes, being one of the possible mechanisms by which oocyte maturation decreased.

\section{Acknowledgements}

The authors wish to thank M.Sc. Alejandro Domínguez for technical assistance, and the slaughterhouse "Los Arcos", State of Mexico for the donation of porcine ovaries.

\section{Authors' contributions}

All authors contributed to the design of the study. FC did the experimental work, statistical analysis, analysis of the results, and wrote the manuscript. YD, $\mathrm{AL}$, and $\mathrm{MB}$ analyzed the results, revised, corrected and approved the manuscript. The authors read and approved the final manuscript.

\section{Funding}

Not applicable.

\section{Availability of data and materials}

Not applicable.

\section{Ethics approval and consent to participate}

This study was performed under the Ethics Committee regulations for the care and use of animals; Metropolitan Autonomous University-Iztapalapa campus

\section{Consent for publication}

Not applicable.

\section{Competing interests}

The authors declare that they have no competing interests to declare.

\section{Author details}

'Departamento de Biología de la Reproducción, División de Ciencias Biológicas y de la Salud, Universidad Autónoma Metropolitana-Iztapalapa, 09340 CDMX, México. ${ }^{2}$ Departamento de Ciencias de la Salud, División de Ciencias Biológicas y de la Salud, Universidad Autónoma Metropolitana-Iztapalapa, 09340 CDMX, México.

Received: 12 September 2020 Accepted: 6 November 2020 Published online: 25 November 2020

\section{References}

1. Brambillasca F, Guglielmo MC, Coticchio G, Renzini MM, Del Canto M, Fadini R. The current challenges to efficient immature oocyte cryopreservation. J Assist Reprod Genet. 2013;30:1531-9.

2. Appeltant R, Somfai T, Santos ECS, Dang-Nguyen TQ, Nagai T, Kikuchi K Effects of vitrification of cumulus-enclosed porcine oocytes at the germinal vesicle stage on cumulus expansion, nuclear progression and cytoplasmic maturation. Reprod Fertil Dev. 2017;29:2419-29.
3. Casillas F, Teteltitla-Silvestre M, Ducolomb Y, Lemus AE, Salazar Z, Casas E, et al. Co-culture with granulosa cells improve the in vitro maturation ability of porcine immature oocytes vitrified with Cryolock. Cryobiology. 2014;69:299-304.

4. Casillas F, Ducolomb Y, Lemus AE, Cuello C, Betancourt M. Porcine embryo production following in vitro fertilization and intracytoplasmic sperm injection from vitrified immature oocytes matured with a granulosa cell coculture system. Cryobiology. 2015;71:299-305.

5. Casillas F, Betancourt M, Cuello C, Ducolomb Y, López A, Juárez-Rojas L, et al. An efficiency comparison of different in vitro fertilization methods: IVF, ICSI and PICSI for embryo development to the blastocyst stage from vitrified porcine immature oocytes. Porcine Health Manag. 2018;4:16.

6. Somfai T, Nguyen HT, Nguyen MT, Dang-Nguyen TQ, Kaneko H, Noguchi J, et al. Vitrification of porcine cumulus-oocyte complexes at the germinal vesicle stage does not trigger apoptosis in oocytes and early embryos, but activates anti-apoptotic BCl-XL gene expression beyond the 4-cell stage. J Reprod Dev. 2020;66:115-23.

7. Somfai T, Yoshioka K, Tanihara F, Kaneko H, Noguchi J, Kashiwazaki N, et al. Generation of live piglets from cryopreserved oocytes for the first time using a defined system for in vitro embryo production. PLoS One. 2014:9:e97731.

8. Nagashima H, Hiruma K, Saito H, Tomii R, Ueno S, Nakayama N, et al. Production of live piglets following cryopreservation of embryos derived form in vitro matured oocytes. Biol Reprod. 2007;76:900-5.

9. Maehara M, Matsunari H, Honda K, Nakano K, Takeuchi Y, Kanai T, et al. Hollow fiber vitrification provides a novel method for cryopreserving in vitro maturation/ in vitro fertilization derived porcine embryos. Biol Reprod. 2012;87:1-8.

10. Alam MH, Miyano T. Interaction between growing oocytes and granulosa cells in vitro. Rep Med Biol. 2020;19:13-23.

11. Pan B, Li J. The art of oocyte meiotic arrest regulation. Rep Biol Endocrinol. 2019;17:1-12.

12. Segretain D, Falk MM. Regulation of connexin biosynthesis, assembly, gap junction formation, and removal. Biochem Biophys Acta. 1662;2004:3-21.

13. Sohl G, Willecke K. Gap junctions and the connexin protein family. Cardiovasc Res. 2004;62:228-32.

14. Sasseville M, Gagnon MC, Guillemette C, Sullivan R, Gilchrist RB, Richard FJ. Regulation of gap junctions in porcine cumulus-oocyte complexes: contributions of granulosa cell contact, gonadotropins, and lipid rafts. Mol Endocrinol. 2009;23:700-10.

15. Norris RP, Freudzon M, Mehlmann LM, Cowan AE, Simon AM, Paul DL, et al. Luteinizing hormone causes MAPK-dependent phosphorylation and closure of Cx43 gap junctions in mouse ovarian follicles: one of two paths to meiotic resumption. Development. 2008;135:3229-38.

16. Santiquet NW, Develle Y, Laroche A, Robert C, Richard FJ. Regulation of gapjunctional communication between cumulus cells during in vitro maturation in swine, a gap-FRAP study. Biol Reprod. 2012;87:46.

17. Domínguez A, Salazar Z, Arenas E, Betancourt M, Ducolomb Y, GonzálezMárquez $\mathrm{H}$, et al. Effect of perfluorooctane sulfonate on viability, maturation and gap junctional intercellular communication of porcine oocytes in vitro. Toxicol in Vitro. 2016;35:93-9.

18. Domínguez A, Salazar Z, Betancourt M, Ducolomb Y, Casas E, Fernández F, et al. Effect of perfluorodecanoic acid on pig oocyte viability, intracellular calcium levels and gap junction intercellular communication during oocyte maturation in vitro. Toxicol in Vitro. 2019:58:224-9.

19. Luciano AM, Franciosi F, Modina SC, Lodde V. Gap junction-mediated communications regulate chromatin remodeling during bovine oocyte growth and differentiation through cAMP-dependent mechanism(s). Biol Reprod. 2011:85:1252-9.

20. Sánchez-Osorio J, Cuello C, Gil MA, Parrilla I, Maside C, Almiñana C, et al. Vitrification and warming of in vivo- derived porcine embryos in a chemically defined medium. Theriogenology. 2010;73:300-8.

21. Thomas RE, Armstrong DT, Gilchrist RB. Bovine cumulus cell-oocyte gap junctional communication during in vitro maturation in response to manipulation of cellspecific cyclic adenosine 3',5'-monophosophate levels. Biol Reprod. 2004;70:548-56.

22. Fernández-Reyes F, Ducolomb Y, Romo S, Casas E, Salazar Z, Betancourt M. Viability, maturation and embryo development in vitro of vitrified immature and porcine oocytes. Cryobiology. 2012;64:261-6.

23. Kang JT, Atikuzzaman M, Kwon DK, Park SJ, Sj K, Moon JH, et al. Developmental competence of porcine oocytes in vitro maturation and in vitro culture under different oxygen concentrations. Zygote. 2012;20:1-8.

24. Khazaei M, Aghaz F. Reactive oxygen species generation and use of antioxidants during in vitro maturation of oocytes. Int J Fertil Steril. 2017;11: $63-70$. 
25. Somfai T, Ozawa M, Noguchi J, Kaneko H, Karja NWK, Farhudin M, et al. Developmental competence of in vitro-fertilized porcine oocytes after in vitro maturation and solid surface vitrification: effect of cryopreservation on oocyte antioxidative system and cell cycle stage. Cryobiology. 2007;55: $115-26$

26. Rojas C, Palom MJ, Albarracin JL, Mogas T. Vitrification of immature and in vitro matured pig oocytes: study of distribution of chromosomes, microtubules, and actin microfilaments. Cryobiology. 2004;49:211-20.

27. Wu C, Rui R, Dai J, Zhang C, Ju S, Xiao-Lu BX, et al. Effects of cryopreservation on the developmental competence, ultrastructure and cytoskeletal structure of porcine oocytes. Mol Rep Dev. 2006;73:1454-62

28. Luciano AM, Chigioni S, Lodde V, Franciosi F, Luvoni GC, Modina SC. Effect of different cryopreservation protocols on cytoskeleton and gap junction mediated communication integrity in feline germinal vesicle stage oocytes. Cryobiology. 2009;59:90-5.

\section{Publisher's Note}

Springer Nature remains neutral with regard to jurisdictional claims in published maps and institutional affiliations.

Ready to submit your research? Choose BMC and benefit from:

- fast, convenient online submission

- thorough peer review by experienced researchers in your field

- rapid publication on acceptance

- support for research data, including large and complex data types

- gold Open Access which fosters wider collaboration and increased citations

- maximum visibility for your research: over $100 \mathrm{M}$ website views per year

At BMC, research is always in progress.

Learn more biomedcentral.com/submissions 